\title{
Bone morphogenetic protein-2 enhances the osteogenic differentiation capacity of mesenchymal stromal cells derived from human bone marrow and umbilical cord
}

\author{
KULISARA MARUPANTHORN $^{1}$, CHAIRAT TANTRAWATPAN ${ }^{1,2}$, PAKPOOM KHEOLAMAI ${ }^{1,2}$, \\ DUANGRAT TANTIKANLAYAPORN ${ }^{1,2}$ and SIRIKUL MANOCHANTR ${ }^{1,2}$ \\ ${ }^{1}$ Division of Cell Biology, Department of Preclinical Sciences, Faculty of Medicine, Thammasat University; \\ ${ }^{2}$ Center of Excellence in Stem Cell Research, Thammasat University, Pathum Thani 12120, Thailand
}

Received February 17, 2016; Accepted January 24, 2017

DOI: $10.3892 /$ ijmm.2017.2872

\begin{abstract}
Mesenchymal stromal cells (MSCs) are multipotent cells that can give rise to different cell types of the mesodermal lineages. They are powerful sources for cell therapy in regenerative medicine as they can be isolated from various tissues, and can be expanded and induced to differentiate into multiple lineages. Recently, the umbilical cord has been suggested as an alternative source of MSCs. Although MSCs derived from the umbilical cord can be induced to differentiate into osteoblasts with a phenotypic similarity to that of bone marrow-derived MSCs, the differentiation ability is not consistent. In addition, MSCs from the umbilical cord require a longer period of time to differentiate into osteoblasts. Previous studies have demonstrated the benefits of bone morphogenetic protein-2 (BMP-2) in bone tissue regeneration. In addition, several studies have supported the use of BMP-2 in periodontal regeneration, sinus lift bone-grafting and non-unions in oral surgery. Although the use of BMP-2 for bone tissue regeneration has been extensively investigated, the BMP-2-induced osteogenic differentiation of MSCs derived from the umbilical cord has not yet been fully examined. Therefore, in this study, we aimed to examine the effects of BMP-2 on the osteogenic differentiation of MSCs derived from umbilical cord compared to that of MSCs derived from bone marrow. The degree of osteogenic differentiation following BMP-2 treatment was determined by assessing alkaline phosphatase (ALP) activity, and the expression profiles of osteogenic differentiation marker genes, osterix $(O s x)$, Runtrelated transcription factor $2($ Run $x 2)$ and osteocalcin $(O c n)$. The results revealed that BMP-2 enhanced the osteogenic differentiation capacity of MSCs derived from both bone marrow and umbilical cord as demonstrated by increased
\end{abstract}

Correspondence to: Dr Sirikul Manochantr, Division of Cell Biology, Department of Preclinical Sciences, Faculty of Medicine, Thammasat University, Paholyotin Road, Klongluang, Pathumthani 12120, Thailand E-mail: bsirikul@gmail.com

Key words: mesenchymal stromal cells, osteogenic differentiation, umbilical cord, bone marrow, bone morphogenetic protein-2
ALP activity and the upregulation of osteogenic differentiation marker genes. The enhancement of the osteogenic differentiation capacity of MSCs by BMP-2 suggests that these MSCs may be used as alternative sources for bone engineering or cell therapy in regenerative medicine.

\section{Introduction}

Currently the number of patients suffering from degenerative bone diseases, such as osteoporosis and osteoarthritis is increasing due to the aging population (1). In addition, fracture non-union remains a clinically important issue. Despite substantial advances in orthopedic surgery, some fractures fail to heal effectively and result in either delayed union or non-union, causing morbidity, prolonged hospitalization and increased expenses. To address these issues, several interventions, including bone autografts have been exploited to improve the capability of bone tissue regeneration, leading to the reduction of both the total costs and hospitalization period (2). However, the use of autografts is associated with certain major issues, including donor site morbidity and limited availability (3). Advances in stem cell research have prompted the development of cell-based therapy for bone repair and the treatment of bone diseases $(4,5)$. Over the past 10 years, tissue engineering using mesenchymal stromal cells (MSCs) has gained increasing attention in regenerative medicine, due to the ability of these cells to differentiate not only into cells of the mesodermal lineage, but also into endodermal and ectodermal lineages $(6,7)$. The characteristics of differentiation in a tissue-specific manner hold great promise for the use of MSCs in the field of regenerative medicine. MSC therapy has been considered to be a potential treatment for several bone diseases $(2,8)$. The use of MSCs for fracture repair has been successfully examined using animal models (9). A previous study reported that autologous bone marrow grafting is an effective and safe method for treating an atrophic tibial diaphyseal non-union (10). Although the progress in the process of osteogenic differentiation of MSCs has been reported, the restricted quantity and quality of functional osteocytes are the main obstacles for the therapeutic application. The identification of novel agents that are able to enhance the osteogenic differentiation capacity of MSCs is thus necessary. 
During normal fracture healing, undifferentiated MSCs, with the aid of bone morphogenetic proteins (BMPs) and regulatory cytokines, proliferate, and differentiate into chondrocytes and osteoblasts, and form bone, thereby repairing the injury (11). A previous study reported that BMP-2 can stimulate the entire process of osteogenic differentiation of bone marrow-derived MSCs (BM-MSCs) in vitro (12). Clinical orthopedic studies have shown the benefits of BMP-2 in bone tissue regeneration. In addition, some studies have supported the use of BMP-2 in periodontal regeneration, sinus lift bone-grafting, and nonunions in bone surgery $(13,14)$. Although MSCs derived from different sources have been assumed to exhibit similar characteristics to MSCs derived from bone marrow, some differences at least in terms of the osteogenic differentiation ability have been reported. MSCs derived from the umbilical cord can be differentiated into osteoblasts with a phenotypic similarity to that of BM-MSCs; however, the differentiation ability is not consistent. In addition, MSCs from the umbilical cord require a longer period of time to differentiate into osteoblasts (15). Although the use of BMP-2 for bone tissue regeneration has been extensively investigated (16-18), the BMP-2-induced osteogenic differentiation of MSCs derived from the umbilical cord has not been fully examined, in particular as regards the underlying molecular events governing osteogenic differentiation.

Thus, in this study, we aimed to examine the effect of BMP-2 on the osteogenic differentiation of MSCs derived from umbilical cord compared to that of MSCs derived from bone marrow. The underlining mechanisms, such as the expression of alkaline phosphatase (ALP) and the changes in the expression of transcription factors involved in the BMP-2-induced osteogenic differentiation of these MSCs were also examined. Our data provide new insight into the effects of BMP-2 on the osteogenic differentiation of MSCs derived from bone marrow and umbilical cord, which may lead to the development of advance techniques for bone tissue regeneration in the future. Our findings also indicate the potential for using these MSCs as alternative sources for bone engineering or cell therapy in regenerative medicine.

\section{Materials and methods}

Cell isolation and culture. The present study was approved by the Human Ethics Committee of Thammasat University No. 1 (Faculty of Medicine; MTU-EC-DS-1-061-57). All subjects participated in the study after providing written informed consent. Bone marrow (BM) was aspirated from healthy volunteers $(n=5)$. Mononuclear cells (MNCs) were isolated using Ficoll-Hypaque solution. BM-MNCs were then cultured in Dulbecco's modified Eagle's medium (DMEM; Gibco-BRL, Carlsbad, CA, USA) supplemented with $10 \%$ fetal bovine serum (FBS; Invitrogen, Carlsbad, CA, USA), $2 \mathrm{mM} \mathrm{L}$-glutamine, $100 \mathrm{U} / \mathrm{ml}$ penicillin and $100 \mu \mathrm{g} / \mathrm{ml}$ streptomycin (both from Gibco-BRL). The medium was changed every 3-4 days. The plastic adherent fibroblast-like cells or so-called BM-MSCs (approximately 80-90\% confluence) were sub-cultured using $0.25 \%$ trypsin-EDTA (Gibco-BRL) and replated at density of $1 \times 10^{4} \mathrm{cell} / \mathrm{cm}^{2}$ for further expansion.

The umbilical cord tissues from full-term pregnancies $(\mathrm{n}=5)$ were minced into small sections and digested with $1.6 \mathrm{mg} /$ $\mathrm{ml}$ collagenase XI and $200 \mathrm{mg} / \mathrm{ml}$ deoxyribonuclease I (both from Sigma-Aldrich, St. Louis, MO, USA) for $4 \mathrm{~h}$ at $37^{\circ} \mathrm{C}$. Subsequently, the cells were washed twice with washing buffer and cultured in complete medium containing DMEM supplemented with FBS, $2 \mathrm{mM}$ L-glutamine, $100 \mathrm{U} / \mathrm{ml}$ penicillin and $100 \mu \mathrm{g} / \mathrm{ml}$ streptomycin. The medium was changed every 3-4 days and non-adherent cells were removed. The adherent cells were further cultured under the same conditions until obtaining the colonies of fibroblast-like cells. For further expansion, the cells were sub-cultured using $0.25 \%$ trypsinEDTA and replated at density of $1 \times 10^{4}$ cells/ $/ \mathrm{cm}^{2}$.

Characterization of MSCs using flow cytometry. The MSCs derived from bone marrow and umbilical cord were trypsinized and re-suspended with phosphate-buffered saline (PBS). In each sample, $5 \times 10^{5}$ MSCs in $50 \mu 1$ of PBS were incubated with $2.5 \mu \mathrm{l}$ of fluorescein isothiocyanate (FITC) or phycoerythrin (PE) conjugated antibodies against human antigens, including FITC-CD45 antibody (Cat. no. 304006), FITC-CD90 antibody (Cat. no. 328108) (both from BioLegend, San Diego, CA, USA), PE-CD105 antibody (Cat. no. 560839; BD Bioscience, Franklin Lakes, NJ, USA), PE-CD34 (Cat. no. 343506) antibody and PE-CD73 antibody (Cat. no. 344004) (both from BioLegend) for $30 \mathrm{~min}$ at $4^{\circ} \mathrm{C}$ in the dark. After washing with PBS, the cells were fixed with $1 \%$ paraformaldehyde in PBS. At least 10,000 labeled cells were acquired and analyzed using a flow cytometer (FACSCalibur $^{\mathrm{TM}}$ ) and CellQuest ${ }^{\circledR}$ software (both from Becton-Dickinson, Franklin Lakes, NJ, USA)

Osteogenic and adipogenic differentiation assay. For osteogenic differentiation, the MSCs were seeded in $35-\mathrm{mm}^{2}$ dishes at a density of $5 \times 10^{3}$ cells $/ \mathrm{cm}^{2}$ and allowed to adhere to the dishes overnight. Subsequently, the cells were washed with PBS and $2 \mathrm{ml}$ of osteogenic differentiation medium [DMEM supplemented with $10 \%$ FBS, $100 \mathrm{U} / \mathrm{ml}$ penicillin, $100 \mu \mathrm{g} / \mathrm{ml}$ streptomycin, $0.5 \mathrm{mM}$ isobutyl-methylxanthine, $0.1 \mu \mathrm{M}$ dexamethasone, $50 \mu \mathrm{g} / \mathrm{ml}$ ascorbic acid (all from Sigma-Aldrich)] was added. On day 7 of culture, $10 \mu \mathrm{M}$ $\beta$-glycerophosphate (Sigma-Aldrich), was added. On day 21 of culture, the cells were harvested for Alizarin Red S staining (Sigma-Aldrich) to determine bone matrix mineralization.

For adipogenic differentiation, the MSCs were cultured with adipogenic differentiation medium [DMEM supplemented with $10 \% \mathrm{FBS}, 100 \mathrm{U} / \mathrm{ml}$ penicillin, $100 \mu \mathrm{g} / \mathrm{ml}$ streptomycin, $0.5 \mathrm{mM}$ isobutyl-methylxanthine, $1 \mu \mathrm{M}$ dexamethasone, $10 \mu \mathrm{M}$ insulin, $100 \mu \mathrm{M}$ indomethacin (all from Sigma-Aldrich)] at a density of $7.8 \times 10^{2}$ cells $/ \mathrm{cm}^{2}$. The medium was changed every 4 days. On day 21 of culture, the cells were fixed in $10 \%$ buffered formalin for $30 \mathrm{~min}$ at room temperature, washed with PBS and incubated with 2\% Oil Red O (Sigma-Aldrich) for $1 \mathrm{~h}$. The cells were washed with distilled $\mathrm{H}_{2} \mathrm{O}$ and observed under an inverted microscope (Nikon TS100; Nikon, Tokyo, Japan). Adipogenic cells were visualized as red-stained lipid droplets. Control cultures without the differentiation stimuli were carried out in parallel to the experiments and stained in the same manner.

Treatment of MSCs with BMP-2. To examine the effect of BMP-2 on the osteogenic differentiation of MSCs, $9.5 \times 10^{3}$ MSCs at passage 4 were cultured in 24-well plate (Corning, Inc., Corning, NY, USA) with osteogenic differentiation medium supplemented with $100 \mathrm{ng} / \mathrm{ml}$ of BMP-2 (R\&D Systems, Minneapolis, MN, USA). MSCs cultured with MSC complete medium and osteogenic differentiation medium were used as controls. Following 
Table I. Sequences of the primers used for PCR and the product size.

\begin{tabular}{lllc}
\hline Gene & \multicolumn{1}{c}{ Forward primer } & \multicolumn{1}{c}{ Reverse primer } & Product size (bp) \\
\hline Runx 2 & 5'-GACAGCCCCAACTTCCTGT-3' & 5'-CCGGAGCTCAGCAGAATAAT-3' & 159 \\
Osterix & 5'-TGCTTGAGGAGGAAGTTCAC-3' & 5'-CTGCTTTGCCCAGAGTTGTT-3' & 114 \\
Osteocalcin & 5'-CTCACACTCCTCGCCCTATT-3' & 5'-TCAGCCAACTCGTCACAGTC-3' & 245 \\
GAPDH & 5'-CAATGACCCCTTCATTGACC-3' & 5'-TTGATTTTGGAGGGATCTCG-3' & 159 \\
\hline
\end{tabular}

incubation for 3, 7, 14, 21 and 28 days, the cell differentiation was measured by qualitative cytochemical staining for ALP using 5-bromo-4-chloro-3-indolylphosphate/nitro blue tetrazolium (BCIP/NBT; Sigma-Aldrich) as a substrate.

ALP activity assay. For quantitative ALP activity, the MSCs were cultured with osteogenic differentiation medium supplemented with $100 \mathrm{ng} / \mathrm{ml}$ of BMP-2 for 3, 7, 14, 21 and 28 days. MSCs cultured with MSC complete medium and osteogenic differentiation medium were used as controls. The colorimetric ALP activity assay was performed using the SensoLyte ${ }^{\circledast N P P}$ Alkaline Phosphatase assay kit (AnaSpec, Inc., Fremont, CA, USA) according to the manufacturer's instructions. Briefly, the cells were washed twice and permeabilized with $0.2 \%$ Triton X-100. Subsequently, the cells were incubated with p-nitrophenyl phosphate (pNPP) chromogenic substrate solution for $45 \mathrm{~min}$ at room temperature. The final solution yielded a yellow-colored product. The stop solution was added and the absorbance was measured at $405 \mathrm{~nm}$ using a microplate reader (BioTex, Winooski, VT, USA). The total protein concentration $(\mathrm{mg} / \mathrm{ml})$ was determined biochemically using the bicinchoninic acid (BCA) assay kit (Sigma-Aldrich). A standard curve was prepared using bovine serum albumin (BSA). ALP levels were normalized against the total protein content. The measured ALP activity was expressed as $\mathrm{ng} / \mathrm{mg}$ protein.

Gene expression analysis by reverse transcription-quantitative $P C R(R T-q P C R)$. To examine the expression of osteogenic differentiation markers following treatment with BMP-2, total RNA was extracted from the MSCs cultured with osteogenic differentiation medium supplemented with $100 \mathrm{ng} / \mathrm{ml}$ of BMP-2 for $3,7,14,21$ and 28 days using TRIzol ${ }^{\circledR}$ reagent (Invitrogen). Two micrograms of total RNA were reverse transcribed into cDNA using SuperScript ${ }^{\circledR}$ III Reverse Transcriptase (Invitrogen). The synthesized cDNAs were subjected to qPCR using the ABI 7500 Real-time PCR system (Applied Biosystems, Foster City, CA, USA). The PCR conditions were as follows: initial denaturation at $95^{\circ} \mathrm{C}$ for $10 \mathrm{~min}$, followed by 40 cycles of denaturation at $95^{\circ} \mathrm{C}$ for $10 \mathrm{sec}$, annealing at $60^{\circ} \mathrm{C}$ for $10 \mathrm{sec}$, and extension at $72^{\circ} \mathrm{C}$ for $40 \mathrm{sec}$. Finally, all PCR product quantifications were normalized to the endogenous control gene, glyceraldehyde3-phosphate dehydrogenase $(G A P D H)$ using the StepOne ${ }^{\mathrm{TM}}$ Software version 2.2 (Applied Biosystems) and presented as the relative mRNA expression level. MSCs cultured with MSC complete medium and osteogenic differentiation medium were used as controls. The primer sequences are listed in Table I.

Statistical analysis. The data were presented as the means \pm standard error of mean (SEM). Statistical compari- sons were performed using the unpaired t-test. A P-value $<0.05$ was considered to indicate a statistically significant difference.

\section{Results}

Characteristic of the MSCs. The cells isolated from bone marrow (BM-MSCs) and umbilical cord (UC-MSCs) were cultured in DMEM supplemented with $10 \%$ FBS. Similar to the BM-MSCs, the cultured UC-MSCs exhibited a spindleshaped morphology with a high nuclear to cytoplasmic ratio following culture for 7 days (Fig. 1A and C). The culture medium was changed every 3 days and the non-adherent cells were removed. Further passages were carried out whenever the cell density reached $90 \%$ confluence, mostly on day 3 following sub-culture. There was no visible difference in the morphology of the UC-MSCs and BM-MSCs (Fig. 1B and D).

For immunophenotyping, both the UC-MSCs and BM-MSCs expressed typical surface markers associated with MSCs, including CD73, CD90 and CD105, and did not express the hematopoietic cell markers, CD34 and CD45. There was no statistically significant difference in the expression of MSC surface markers between the UC-MSCs and BM-MSCs (Fig. 2).

To examine the differentiation potential, the UC-MSCs and BM-MSCs were induced to differentiate into adipocytes or osteoblasts by culturing the cells in adipogenic or osteogenic differentiation media. After 3 weeks of adipogenic induction, the BM-MSCs became large cells which contained numerous lipid droplets in their cytoplasm. In contrast to the BM-MSCs, the differentiated UC-MSCs exhibited small amounts of lipid droplets. However, these lipid droplets were positive for Oil Red O staining (Fig. 3B and D). The control cultures did not have any lipid droplets in their cytoplasm and were negative for Oil Red O staining (Fig. 3A and C). For osteogenic differentiation, the mineralized matrix was detected in the BM-MSCs and UC-MSCs following induction for 21 and 28 days (Fig. 4B and D). The control cultures were negative for Alizarin Red staining (Fig. 4A and C). Of note, the MSCs from both sources had the capacity to differentiate into osteoblasts and adipocytes; however, the UC-MSCs required a longer induction period than the BM-MSCs.

Expression of ALP following treatment with BMP-2. To determine the effects of the BMP-2 on the osteogenic differentiation of UC-MSCs in comparison to that of BM-MSCs, the cells were cultured in three different media, complete medium, osteogenic differentiation medium and osteogenic differentiation medium in the presence of BMP-2 for 3, 7, 14, 21 and 28 days. Following induction for 3 days, few ALP-positive cells were observed in the BM-MSCs cultured with osteogenic 


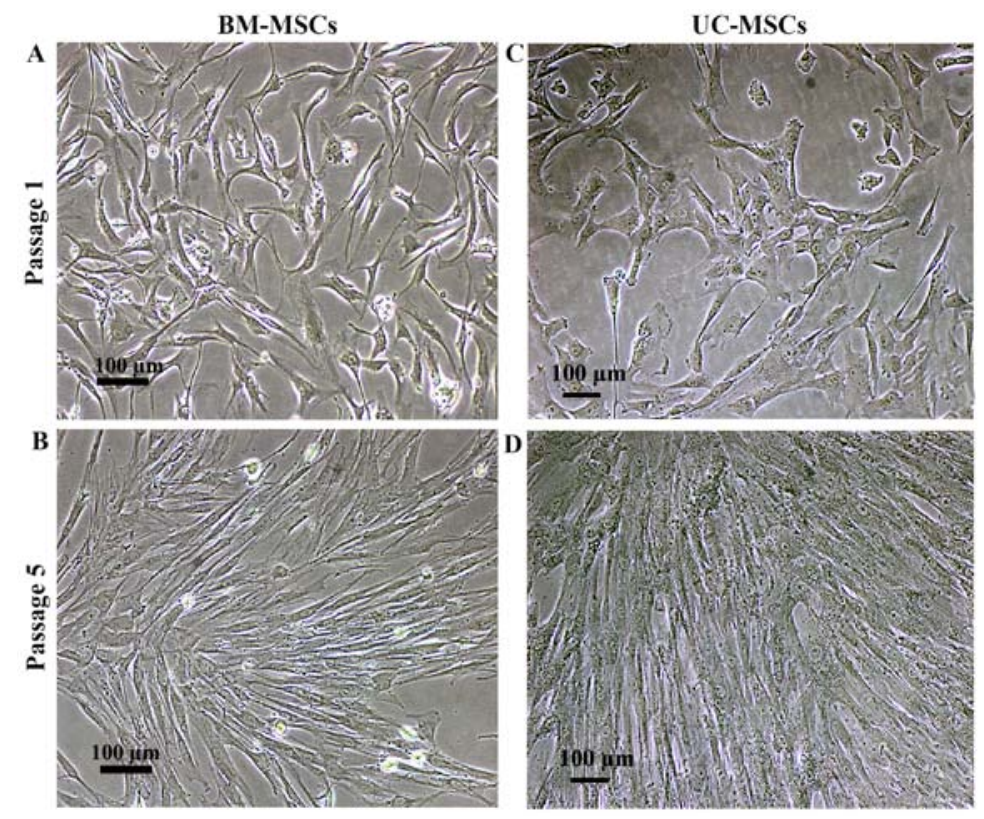

Figure 1. Morphology of mesenchymal stromal cells isolated from (A and B) bone marrow and (C and D) umbilical cord. Scale bar, $100 \mu \mathrm{m}$.
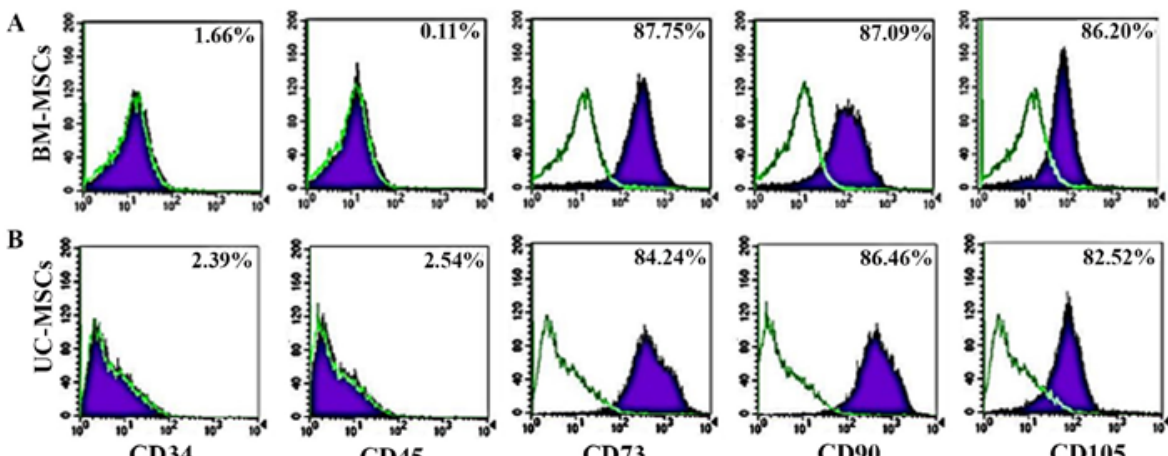

Figure 2. Flow cytometric analysis of the expression of cell surface markers of (A) bone marrow-derived mesenchymal stromal cells (BM-MSCs) and (B) umbilical cord-derived mesenchymal stromal cells (UC-MSCs) at passage 3-5 (B).

differentiation medium in the absence of BMP-2 (Fig. 5A). Of note, treatment with BMP-2 increased the expression of ALP (Fig. 5B). In addition, the UC-MSCs treated with BMP-2 for 3 days exhibited a higher ALP expression than those in the untreated group (Fig. 5C and D). Following sequential culture, the expression of ALP increased over time in both the BM-MSCs and UC-MSCs cultured in osteogenic differentiation medium with or without BMP-2. Remarkably, a moderate number of ALP-positive cells was observed in the BM-MSCs treated with BMP-2 for 14 days (Fig. 5J). The intensity of ALP staining was significantly increased in the BM-MSCs treated with BMP-2 for 21 and 28 days as compared to the BM-MSCs cultured in osteogenic differentiation medium without BMP-2 (Fig. 5M, N, Q and R). In the UC-MSCs, treatment with BMP-2 increased the expression of ALP (Fig. 5H, L, P and T) as compared to the UC-MSCs cultured in osteogenic differentiation medium without BMP-2 (Fig. 5G, K, O and S). However, the intensity of ALP staining was significantly less than that of the BM-MSCs at each time point examined (Fig. 5).

The activity of intracellular ALP in both the BM-MSCs and UC-MSCs was also quantitative assessed on day 3, 7, 14, 21 and 28 in the three groups, namely the negative control
(MSCs cultured in complete medium), untreated group (MSCs cultured in osteogenic differentiation medium), BMP-2 treated group (MSCs cultured in osteogenic differentiation medium supplemented with BMP-2). Of the three ways of treating MSCs, BMP-2 treatment of both the BM-MSCs and UC-MSCs was shown to be clearly superior compared to the other two treatments in regards to increasing ALP activity (Fig. 6). In addition, the activity of ALP in the BMP-2-treated groups significantly increased with time. Similar to ALP staining, although ALP activity increased over time in the BMP-2treated groups, ALP activity in the UC-MSCs was significantly less than that of the BM-MSCs at each time point examined.

Effect of BMP-2 on the expression levels of osteogenic lineage genes. In this study, the effect of BMP-2 on the osteogenic differentiation of BM-MSCs and UC-MSCs was further investigated through gene expression analysis of Runt-related transcription factor 2 (Runx2), osterix (Osx) and osteocalcin (Ocn) following 3, 7, 14, 21 and 28 days of culture. The results revealed that in the BM-MSCs, BMP-2 significantly upregulated the expression of the osteogenic lineage genes, Run $x 2$, Os $x$ and Ocn on days 7, 14, 21 and 28 following osteogenic induction, 

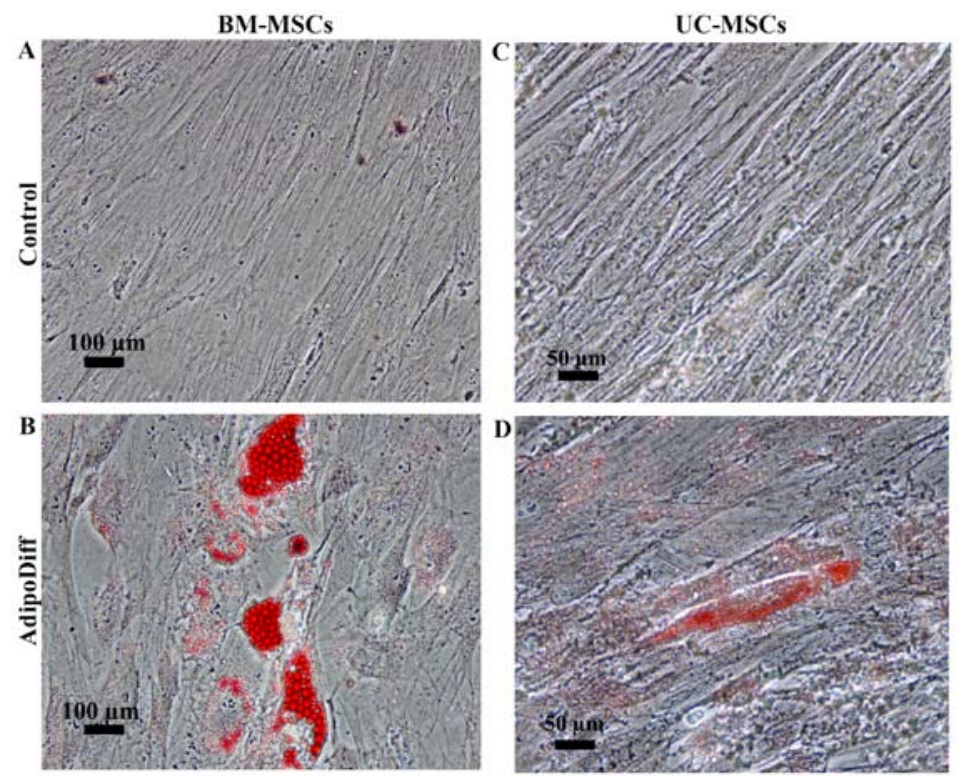

Figure 3. Adipogenic differentiation potential of umbilical cord-derived mesenchymal stromal cells (UC-MSCs) in comparison to bone marrow-derived mesenchymal stromal cells (BM-MSCs). (A) BM-MSCs cultured in DMEM supplemented with 10\% fetal bovine serum (FBS) for 21 days. (B) Oil Red O staining of BM-MSCs cultured in adipogenic differentiation medium for 28 days. (C) UC-MSCs cultured in DMEM supplemented with $10 \%$ FBS for 21 days. (D) Oil Red O staining of UC-MSCs cultured in adipogenic differentiation medium for 28 days. Scale bar, $50 \mu \mathrm{m}$.
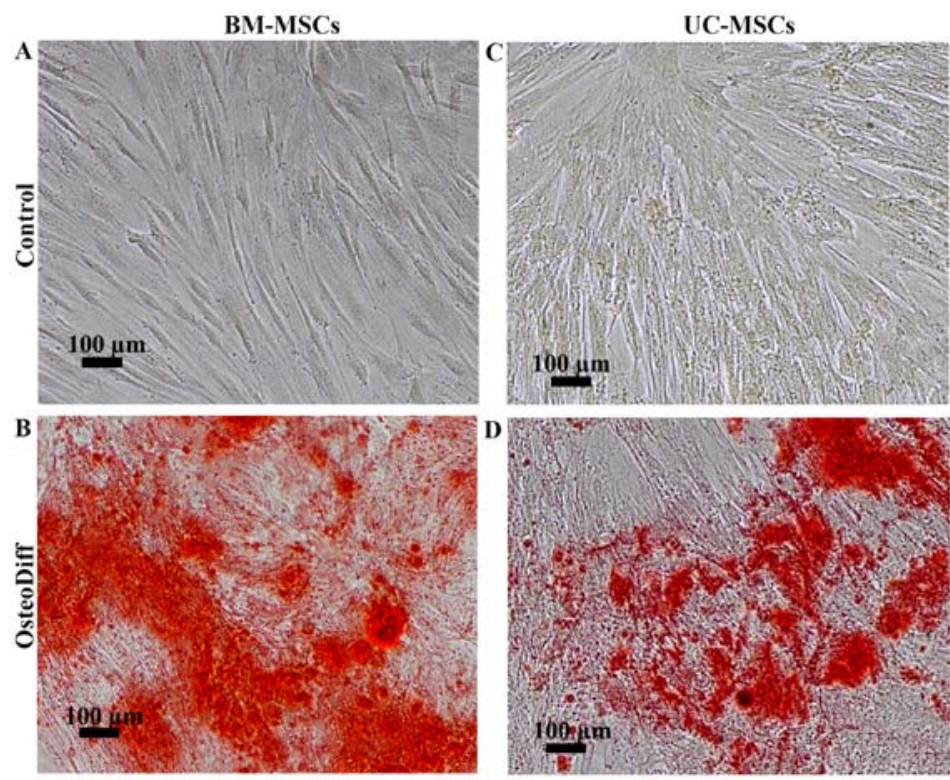

Figure 4. Osteogenic differentiation potential of umbilical cord-derived mesenchymal stromal cells (UC-MSCs) in comparison to bone marrow-derived mesenchymal stromal cells (BM-MSCs). (A) The negative result for Alizarin Red staining of BM-MSCs cultured in DMEM supplemented with $10 \%$ fetal bovine serum (FBS) for 21 days. (B) The positive result for Alizarin Red staining of BM-MSCs cultured in osteogenic differentiation medium for 21 days. (C) The negative result for Alizarin Red staining of UC-MSCs cultured in DMEM supplemented with 10\% FBS for 28 days. (D) The positive result for Alizarin Red staining of UC-MSCs cultured in osteogenic differentiation medium for 28 days. Scale bar, $100 \mu \mathrm{m}$.

while there were no significant differences in the expression levels of these osteogenic lineage genes during the earlier time points (day 3; Fig. 7A, C and E). The expression of Runx2 increased over time from day 3 to 14 in the BM-MSC cultures. The peak in Runx2 mRNA expression was observed on day 14 in the BM-MSCs cultured in osteogenic differentiation medium with or without BMP-2. Nevertheless, the BM-MSCs cultured in osteogenic differentiation with BMP-2 exhibited a significantly higher expression of Run $x 2$ than those cultured in osteogenic differentiation medium without BMP-2 (Fig. 7A).
In contrast to the BM-MSCs, Runx2 mRNA expression increased over time from days 3 to 28 in the UC-MSCs cultured in osteogenic differentiation medium with or without BMP-2. Of note, the UC-MSCs treated with BMP-2 exhibited a significantly higher expression of Runx 2 than those in the untreated group (Fig. 7B). The effect of BMP-2 on the expression levels of other osteogenic lineage genes in the cultured UC-MSCs also differed from that of the BM-MSCs. The mRNA expression of Osx increased over time from day 3 to 28 in the BM-MSCs and UC-MSCs cultured in osteogenic differentiation with or 


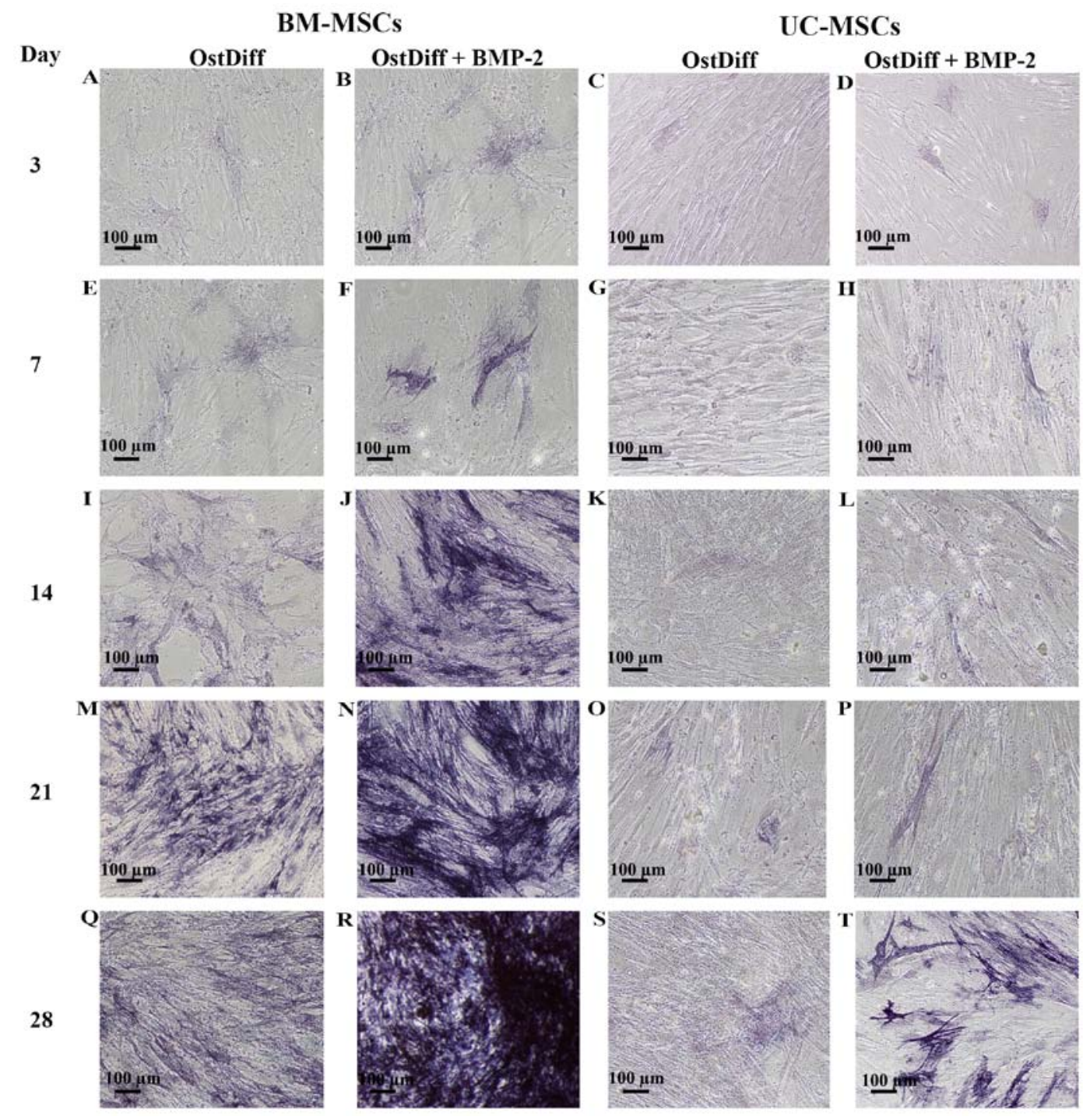

Figure 5. Representative images of alkaline phosphatase (ALP) staining of umbilical cord-derived mesenchymal stromal cells (UC-MSCs) and bone marrowderived mesenchymal stromal cells (BM-MSCs) following treatment with bone morphogenetic protein-2 (BMP-2) for 3, 7, 14, 21 and 28 days. OstDiff, osteogenic differentiation medium; OstDiff + BMP-2, osteogenic differentiation medium supplemented with BMP-2. Scale bar, $100 \mu \mathrm{m}$.

without BMP-2 (Fig. 7C and D). However, BMP-2 significantly upregulated the gene expression in the cultured UC-MSCs on days 14, 21 and 28 of culture (Fig. 7D), while the effect of BMP-2 in upregulating gene expression was observed on days 7 , 14, 21 and 28 in the cultured BM-MSCs (Fig. 7C). Similar to Osx, a minimum Ocn mRNA expression was detected in the BM-MSCs on day 3 and significantly increased Ocn levels were observed in the BM-MSCs treated with BMP-2 compared with the untreated controls on days 7, 14, 21 and 28 (Fig. 7E). The mRNA expression of Ocn was increased to the same extent with time in the UC-MSCs in both the BMP-2-treated and untreated groups (Fig. 7F). However, the increased gene expression of $\mathrm{Ocn}$ in the UC-MSCs was significantly observed in the BMP-2-treated group on days 14, 21 and 28.

\section{Discussion}

MSCs are adherent marrow stromal cells which have a self-renewal ability and the potential to differentiate into osteoblasts, chondrocytes, myoblasts and adipocytes (19). As multipotent progenitors, MSCs have been regarded as ideal seed cells for scientific research and bone tissue engineering $(2,20)$. However, the promotion of MSC differentiation into osteoblasts by osteogenic induction factors is one of the most crucial issues in bone tissue engineering (2,21). BMP-2 is one of the most potent BMPs for promoting the osteogenic differentiation of BM-MSCs both in vitro and in vivo $(22,23)$. A previous study reported that BMP-2-producing cells, via adenoviral gene transfer, produced sufficient protein to heal segmental bone defects in a rat model (24). In addition, the acceleration of bone regeneration using rat BM-MSCs transduced with BMP-2 was higher than that of using MSCs alone (25). Several other animal studies performed with the implantation of autologous BM-MSCs using different scaffolds have resulted in bone regeneration $(9,26)$. Although BM-MSCs are dominant seed cell sources for bone engineering, the limited cell number and the invasive procedure for harvesting cell restrict their use in clinical fields. Over the past few years, UC-MSCs are considered as promising alternative source for MSCs used in the research and application of stem cell therapy as they are easy to be isolated and expanded in vitro and 

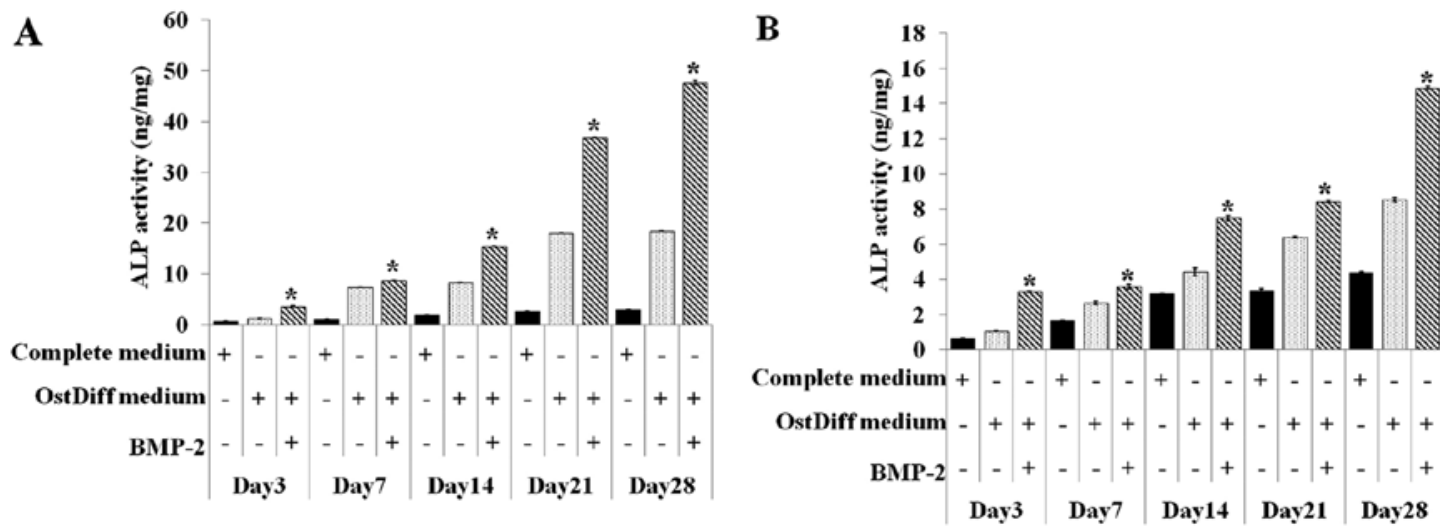

Figure 6. Quantitative evaluation of the osteogenic differentiation capacity of (B) umbilical cord-derived mesenchymal stromal cells (UC-MSCs) (B) in comparison to (A) bone marrow-derived mesenchymal stromal cells (BM-MSCs) using alkaline phosphatase (ALP) activity assay. Results are the summary of triplicate cultures. Three independent experiments were conducted with similar results. ${ }^{*} \mathrm{P}<0.05$ in comparison to osteogenic differentiation medium (OstDiff medium).
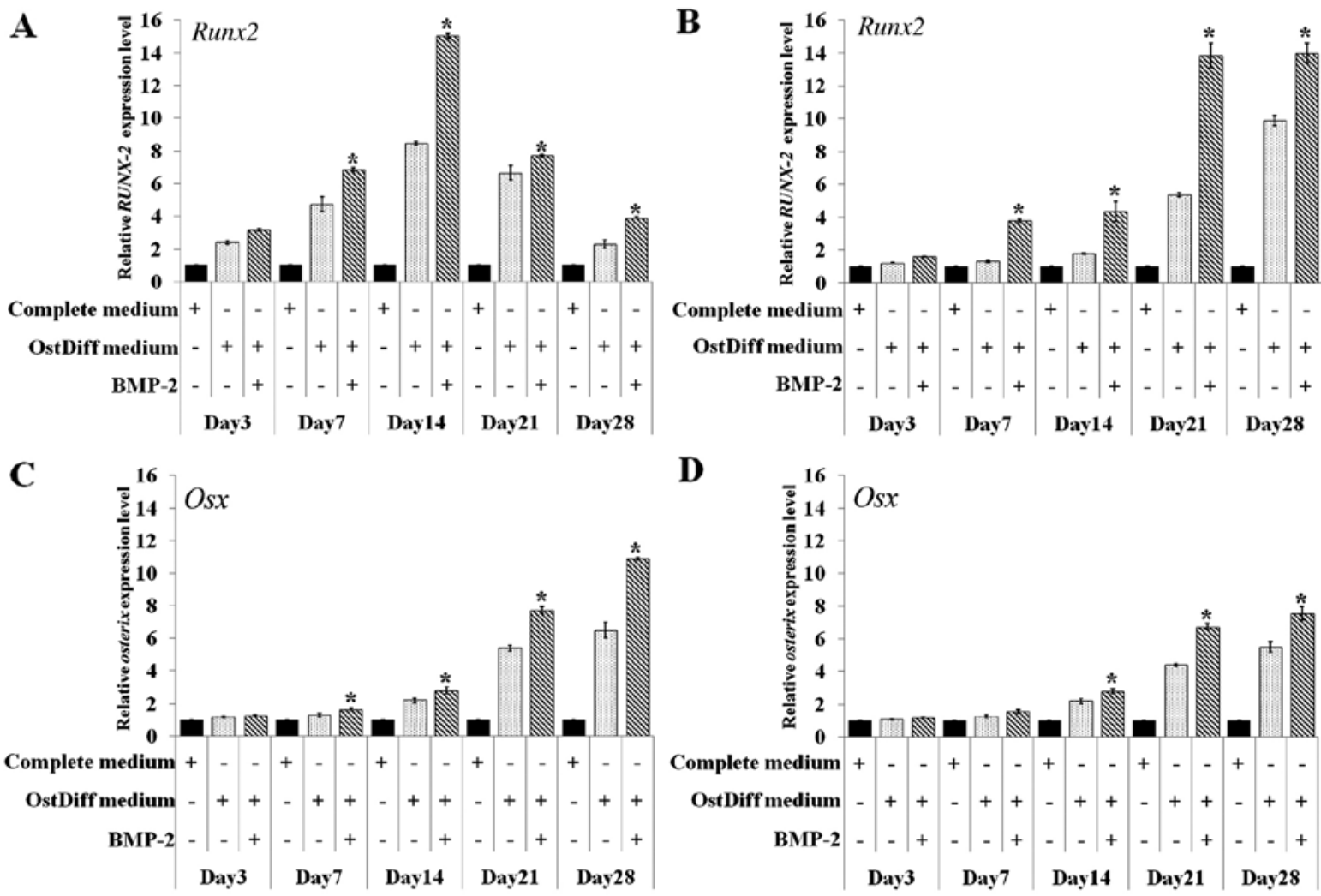

D
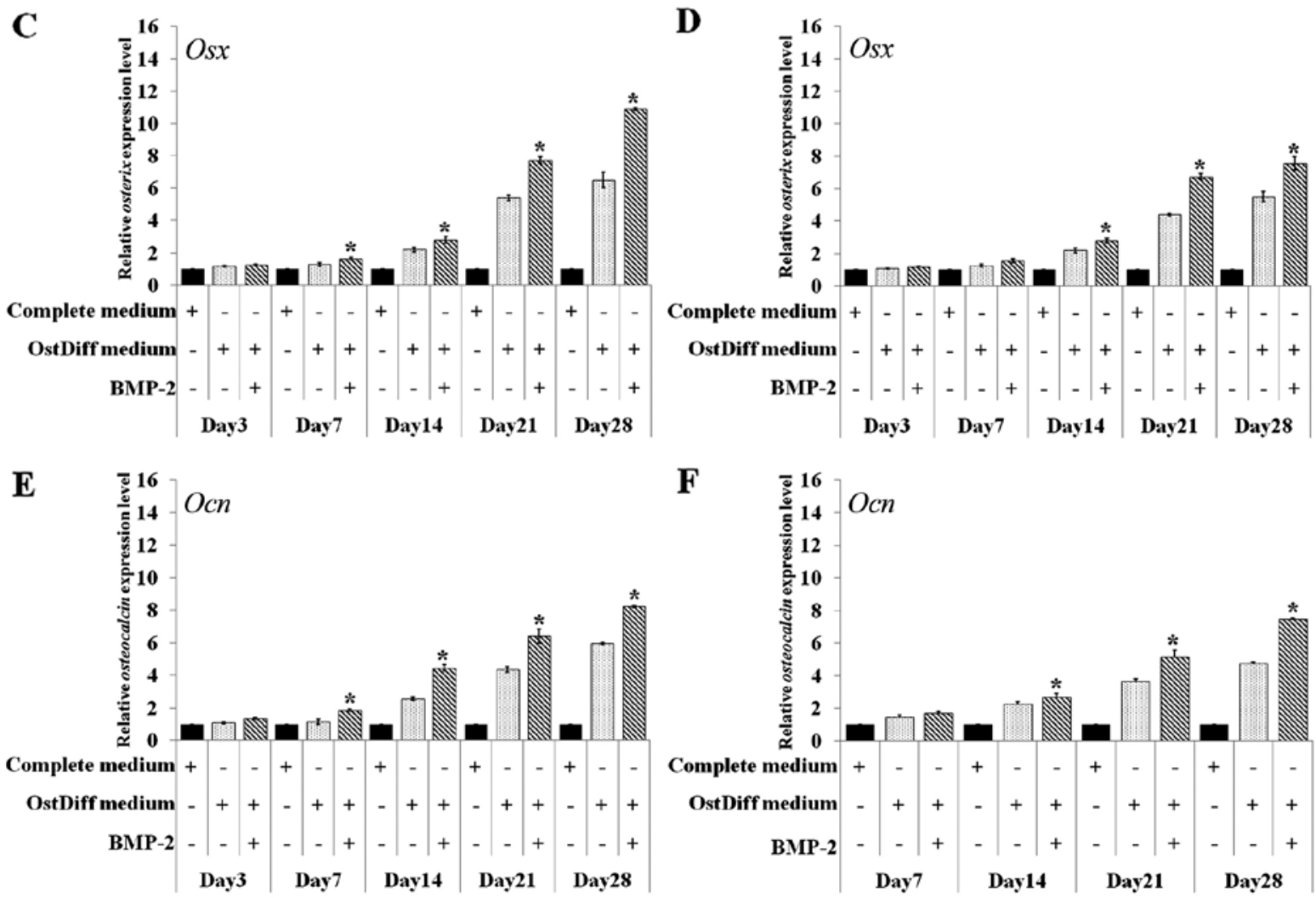

Figure 7. RT-qPCR of the mRNA expression of the osteogenic differentiation marker genes, Runt-related transcription factor 2 (Runx2), osterix (Osx) and osteocalcin $(O c n)$, in (A, C and E) bone marrow-derived mesenchymal stromal cells (BM-MSCs) and (B, D and F) umbilical cord-derived mesenchymal stromal cells (UC-MSCs) following treatment with bone morphogenetic protein-2 (BMP-2) for 3, 7, 14, 21 and 28 days. Data are the representative of 3 independent experiments. ${ }^{*} \mathrm{P}<0.05$ in comparison to osteogenic differentiation medium (OstDiff medium). 
can be attained by a less invasive method without harming the mother or infant $(15,27)$. Although the UC-MSCs share similar characteristics to the BM-MSCs, MSCs from different sources differ in their differentiation potential and gene expression profile (28). There is evidence suggested that UC-MSCS require a longer period of time for osteogenic differentiation compared to BM-MSCs (15). Therefore, it is still a challenge to enhance the osteogenic differentiation potential of UC-MSCs for clinical application. Although studies have demonstrated the benefits of BMP-2 in bone tissue regeneration $(29,30)$, the BMP-2-induced osteogenic differentiation of MSCs derived from the umbilical cord has not been fully examined.

In this study, MSCs were characterized according to the criteria of the International Society for Cellular Therapy (31). The results revealed that the plastic-adherent MSCs isolated from both bone marrow and umbilical cord displayed a fibroblast-like morphology. They were typically negative for CD34, CD45 and were shown to be positive for CD73, CD90 and CD105. The effect of BMP-2 on osteogenic differentiation was investigated to clarify the role of BMP-2 in the function of MSC differentiation. The expression of ALP was monitored using ALP staining and ALP activity assay. ALP is produced by osteoblasts and is hypothesized to be involved in the degradation of inorganic pyrophosphates to provide sufficient local phosphate or inorganic pyrophosphate for the occurrence of mineralization (32). Therefore, ALP activity is commonly used as a marker of osteogenesis to reflect the degree of osteogenic differentiation. Our results revealed that BMP-2 treatment enhanced the efficiency of osteogenic differentiation of both human BM-MSCs and UC-MSCs as evidenced by the increasing level of ALP staining and ALP activity. Accordingly, the expression levels of osteogenic marker genes were also increased by BMP-2 treatment in both human BM-MSCs and UC-MSCs.

Although the molecular mechanisms underlying BMP-2mediated osteogenesis remain to be fully understood, various studies have demonstrated that BMP-2 plays a critical role in the osteogenic differentiation of MSCs $(2,12,23)$. In addition to the direct application of recombinant BMP-2 proteins, it has been confirmed that the adenoviral vector-mediated gene transfer of BMP-2 has the ability to induce bone formation both in vitro and in vivo (13). A previous study using the mouse myoblast cell line, $\mathrm{C} 2 \mathrm{C} 12$, demonstrated that BMP-2 inhibited myogenic differentiation, and instead diverted their differentiation pathway into that of osteoblasts (33). Furthermore, BMP-2 has also been shown to induce Runx2 expression in mesenchymal progenitors in a Smad-dependent manner and to regulate the expression of target genes which are involved in osteoblast differentiation (34).

In this study, we demonstrated that osteogenic signaling molecules, including Runx2, Osx and Ocn were upregulated in the BM-MSCs following treatment with BMP-2. Of note, the peak expression of Runx2 in BM-MSCs cultured with osteogenic differentiation medium in the presence or absence of BMP-2 was observed on day 14. The addition of BMP-2 enhanced the expression of Runx2. In contrast to the BM-MSCs, the highest expression of Runx2 in the UC-MSCS cultured with osteogenic differentiation medium in the presence or absence of BMP-2 was observed on day 28 and BMP-2 enhanced the expression of Runx2 in the UC-MSCs similar to the BM-MSCs.
Runx2 is a pivotal osteogenic transcription factor. It may be one of the earliest master transcription factors that directs the differentiation of MSCs into osteoblasts (35). A previous study reported that Runx2 is expressed in $O s x^{-/-}$mice, while $O s x$ is not expressed in Runx $2^{-/}$mice (36). Based on this finding, Runx2 has been proposed to be an upstream regulator of $O s x$ expression (37). Runx 2 and Osx may control osteogenesis in a manner analogous to the master transcription factors. A previous study suggested that Runx2 plays a role in the commitment step for the common progenitor cells for osteoblasts and chondrocytes, whereas $O s x$ plays a role in the final differentiation step in osteogenesis (36). This notion is further supported by evidence that the expression of $O c n$, the final differentiation marker of osteogenesis, is induced by $O s x$ overexpression but not by Runx2 overexpression (38). These data support the findings of this study. The expression of $O s x$ at each time point overlapped with the expression of Ocn. Of note, BMP-2 treatment significantly enhanced the expression of Osx and Ocn in both the BM-MSCs and UC-MSCs. Although BMP-2 enhanced the osteogenic differentiation capability of UC-MSCs, the effect was less pronounced compared to the BM-MSCs. This may be due to the endogenous difference in the osteogenic differentiation capacity between BM-MSCs and UC-MSCs, as shown by the finding that the endogenous ALP activity of the UC-MSCs was much lower than that of the BM-MSCs cultured under the same conditions. It may be possible that BMP-2 is not the only initial inducer of Runx2 expression during the osteogenic differentiation of UC-MSCs. A previous study reported that some developmentally important ligands, such as transforming growth factor (TGF)- $\beta 1$, fibroblast growth factors (FGFs) and BMP-9, can stimulate Runx2 expression and enhance ALP expression in bone development $(39,40)$. In addition, the suitable concentration of BMP-2 is still an issue as regards the enhancement of the osteogenic capacity of UC-MSCs. Nevertheless, this study demonstrated that BMP-2 treatment enhanced the osteogenic differentiation capacity of both BM-MSCs and UC-MSCs, as evidenced by an increase in ALP expression and osteogenic gene expression.

In conclusion, the results obtained from this study indicate that MSCs derived from the umbilical cord provide an exciting and promising stem cell source for bone repair in skeletal diseases. Our findings demonstrated that BMP-2 enhanced the osteogenic differentiation capacity of both BM-MSCs and UC-MSCs. These MSCs may potentially be used as a therapeutic agent in the treatment of patients with bone defects. The use of MSCs derived from bone marrow and umbilical cord may prove to be an innovative treatment for many musculoskeletal diseases, including fracture non-unions and a number of metabolic bone diseases; therefore, their use in clinical applications warrants rigorous assessment.

\section{Acknowledgements}

The authors would like to thank the staff at the delivery room, Thammasat University Hospital, for facilitating specimen collection and all the volunteers for kindly donating the tissues for this study. This study was supported by grants from Thailand Research Fund and Thammasat University (RSA5980042) and Center of Excellence in Stem Cell Research, Thammasat University. 


\section{References}

1. Loeser RF: Age-related changes in the musculoskeletal system and the development of osteoarthritis. Clin Geriatr Med 26: 371-386, 2010

2. Petite H, Viateau V, Bensaïd W, Meunier A, de Pollak C, Bourguignon M, Oudina K, Sedel L and Guillemin G: Tissueengineered bone regeneration. Nat Biotechnol 18: 959-963, 2000.

3. Stock UA and Vacanti JP: Tissue engineering: current state and prospects. Annu Rev Med 52: 443-451, 2001.

4. Wang XF, Song Y, Liu YS, Sun YC, Wang YG, Wang Y and Lyu PJ: Osteogenic differentiation of three-dimensional bioprinted constructs consisting of human adipose-derived stem cells in vitro and in vivo. PLoS One 11: e0157214, 2016.

5. Yi H, Ur Rehman F, Zhao C, Liu B and He N: Recent advances in nano scaffolds for bone repair. Bone Res 4: 16050, 2016.

6. Krampera M,Pizzolo G, Aprili G and Franchini M: Mesenchymal stem cells for bone, cartilage, tendon and skeletal muscle repair. Bone 39: 678-683, 2006.

7. Krampera M, Marconi S, Pasini A, Galiè M, Rigotti G, Mosna F, Tinelli M, Lovato L, Anghileri E, Andreini A, et al: Induction of neural-like differentiation in human mesenchymal stem cells derived from bone marrow, fat, spleen and thymus. Bone 40 : 382-390, 2007

8. Li X, Ling W, Pennisi A, Wang Y, Khan S, Heidaran M, Pal A, Zhang X, He S, Zeitlin A, et al: Human placenta-derived adherent cells prevent bone loss, stimulate bone formation, and suppress growth of multiple myeloma in bone. Stem Cells 29: 263-273, 2011

9. Arinzeh TL, Peter SJ, Archambault MP, van den Bos C, Gordon S, Kraus K, Smith A and Kadiyala S: Allogeneic mesenchymal stem cells regenerate bone in a critical-sized canine segmental defect. J Bone Joint Surg Am 85-A: 1927-1935, 2003.

10. Hernigou P, Poignard A, Manicom O, Mathieu G and Rouard H: The use of percutaneous autologous bone marrow transplantation in nonunion and avascular necrosis of bone. J Bone Joint Surg $\mathrm{Br}$ 87: 896-902, 2005

11. Tseng SS, Lee MA and Reddi AH: Nonunions and the potential of stem cells in fracture-healing. J Bone Joint Surg Am 90 (Suppl 1): 92-98, 2008.

12. De Biase P and Capanna R: Clinical applications of BMPs. Injury 36 (Suppl 3): S43-S46, 2005.

13. Bais MV, Wigner N, Young M, Toholka R, Graves DT, Morgan EF, Gerstenfeld LC and Einhorn TA: BMP2 is essential for post natal osteogenesis but not for recruitment of osteogenic stem cells. Bone 45: 254-266, 2009.

14. Osyczka AM and Leboy PS: Bone morphogenetic protein regulation of early osteoblast genes in human marrow stromal cells is mediated by extracellular signal-regulated kinase and phosphatidylinositol 3-kinase signaling. Endocrinology 146: 3428-3437, 2005.

15. Manochantr S, U-pratya Y, Kheolamai P, Rojphisan S, Chayosumrit M, Tantrawatpan C, Supokawej A and Issaragrisil S: Immunosuppressive properties of mesenchymal stromal cells derived from amnion, placenta, Wharton's jelly and umbilical cord. Intern Med J 43: 430-439, 2013.

16. Cheng YH, Lin FH, Wang CY, Hsiao CY, Chen HC, Kuo HY Tsai TF and Chiou SH: Recovery of oxidative stress-induced damage in Cisd2-deficient cardiomyocytes by sustained release of ferulic acid from injectable hydrogel. Biomaterials 103: 207-218, 2016

17. Scarfi S: Use of bone morphogenetic proteins in mesenchymal stem cell stimulation of cartilage and bone repair. World J Stem Cells 8: 1-12, 2016.

18. Dang PN, Dwivedi N, Phillips LM, Yu X, Herberg S, Bowerman C, Solorio LD, Murphy WL and Alsberg E: Controlled dual growth factor delivery from microparticles incorporated within human bone marrow-derived mesenchymal stem cell aggregates for enhanced bone tissue engineering via endochondral ossification. Stem Cells Transl Med 5: 206-217, 2016.

19. Pittenger MF, Mackay AM, Beck SC, Jaiswal RK, Douglas R, Mosca JD, Moorman MA, Simonetti DW, Craig S and Marshak DR: Multilineage potential of adult human mesenchymal stem cells. Science 284: 143-147, 1999.

20. Koç ON and Lazarus HM: Mesenchymal stem cells: Heading into the clinic. Bone Marrow Transplant 27: 235-239, 2001.

21. Na K, Kim SW, Sun BK, Woo DG, Yang HN, Chung HM and Park KH: Osteogenic differentiation of rabbit mesenchymal stem cells in thermo-reversible hydrogel constructs containing hydroxyapatite and bone morphogenic protein-2 (BMP-2). Biomaterials 28: 2631-2637, 2007.
22. Luu HH, Song WX, Luo X, Manning D, Luo J, Deng ZL, Sharff KA, Montag AG, Haydon RC and He TC: Distinct roles of bone morphogenetic proteins in osteogenic differentiation of mesenchymal stem cells. J Orthop Res 25: 665-677, 2007.

23. Osyczka AM, Damek-Poprawa M, Wojtowicz A and Akintoye SO Age and skeletal sites affect BMP-2 responsiveness of human bone marrow stromal cells. Connect Tissue Res 50: 270-277, 2009.

24. Lieberman JR, Daluiski A, Stevenson S, Wu L, McAllister P, Lee YP, Kabo JM, Finerman GA, Berk AJ and Witte ON: The effect of regional gene therapy with bone morphogenetic protein-2-producing bone-marrow cells on the repair of segmental femoral defects in rats. J Bone Joint Surg Am 81: 905-917, 1999.

25. Lin Z, Wang JS, Lin L, Zhang J, Liu Y, Shuai M and Li Q: Effects of BMP2 and VEGF165 on the osteogenic differentiation of rat bone marrow-derived mesenchymal stem cells. Exp Ther Med 7: 625-629, 2014.

26. Viateau V1, Guillemin G, Bousson V, Oudina K, Hannouche D, Sedel L, Logeart-Avramoglou D and Petite H: Long-bone critical-size defects treated with tissue-engineered grafts: A study on sheep. J Orthop Res 25: 741-749, 2007.

27. Lu LL, Liu YJ, Yang SG, Zhao QJ, Wang X, Gong W, Han ZB, $\mathrm{Xu} Z \mathrm{ZS}, \mathrm{Lu}$ YX,Liu D, et al: Isolation and characterization of human umbilical cord mesenchymal stem cells with hematopoiesissupportive function and other potentials. Haematologica 91 1017-1026, 2006.

28. Wagner W, Wein F, Seckinger A, Frankhauser M, Wirkner U, Krause U, Blake J, Schwager C, Eckstein V, Ansorge W, et al: Comparative characteristics of mesenchymal stem cells from human bone marrow, adipose tissue, and umbilical cord blood. Exp Hematol 33: 1402-1416, 2005.

29. Kratchmarova I, Blagoev B, Haack-Sorensen M, Kassem M and Mann M: Mechanism of divergent growth factor effects in mesenchymal stem cell differentiation. Science 308: 1472-1477, 2005.

30. Hsiao HY, Yang SR, Brey EM, Chu IM and Cheng MH: Hydrogel delivery of mesenchymal stem cell-expressing bone morphogenetic protein-2 enhances bone defect repair. Plast Reconstr Surg Glob Open 4: e838, 2016.

31. Dominici M,Le Blanc K, Mueller I, Slaper-Cortenbach I, Marini F, Krause D, Deans R, Keating A, Prockop Dj and Horwitz E: Minimal criteria for defining multipotent mesenchymal stromal cells. The International Society for Cellular Therapy position statement. Cytotherapy 8: 315-317, 2006.

32. Mikami Y, Tsuda H, Akiyama Y, Honda M, Shimizu N, Suzuki N and Komiyama K: Alkaline phosphatase determines polyphosphate-induced mineralization in a cell-type independent manner. J Bone Miner Metab 34: 627-637, 2016.

33. Katagiri T, Yamaguchi A, Komaki M, Abe E, Takahashi N, Ikeda T, Rosen V, Wozney JM, Fujisawa-Sehara A and Suda T: Bone morphogenetic protein-2 converts the differentiation pathway of C2C12 myoblasts into the osteoblast lineage. J Cell Biol 127: 1755-1766, 1994.

34. Nishimura R, Hata K, Ikeda F, Ichida F, Shimoyama A, Matsubara T, Wada M, Amano K and Yoneda T: Signal transduction and transcriptional regulation during mesenchymal cell differentiation. J Bone Miner Metab 26: 203-212, 2008.

35. Ryoo HM, Lee MH and Kim YJ: Critical molecular switches involved in BMP-2-induced osteogenic differentiation of mesenchymal cells. Gene 366: 51-57, 2006.

36. Nakashima K, Zhou X, Kunkel G, Zhang Z, Deng JM, Behringer RR and de Crombrugghe $B$ : The novel zinc fingercontaining transcription factor osterix is required for osteoblast differentiation and bone formation. Cell 108: 17-29, 2002

37. Tsao YT, Huang YJ, Wu HH, Liu YA, Liu YS and Lee OK: Osteocalcin mediates biomineralization during osteogenic maturation in human mesenchymal stromal cells. Int J Mol Sci 18: 159, 2017.

38. Lee KS, Kim HJ, Li QL, Chi XZ, Ueta C, Komori T, Wozney JM, Kim EG, Choi JY, Ryoo HM and Bae SC: Runx2 is a common target of transforming growth factor betal and bone morphogenetic protein 2 , and cooperation between Runx 2 and Smad5 induces osteoblast-specific gene expression in the pluripotent mesenchymal precursor cell line C2C12. Mol Cell Biol 20: 8783$8792,2000$.

39. Kim HJ, Kim JH, Bae SC, Choi JY, Kim HJ and Ryoo HM: The protein kinase $C$ pathway plays a central role in the fibroblast growth factor-stimulated expression and transactivation activity of Runx2. J Biol Chem 278: 319-326, 2003.

40. Kim YJ, Lee MH, Wozney JM, Cho JY and Ryoo HM: Bone morphogenetic protein-2-induced alkaline phosphatase expression is stimulated by Dlx 5 and repressed by Msx2. J Biol Chem 279: 50773-50780, 2004. 